\title{
Occult Filum terminale
}

California, Davis., 2916 Stockton Blvd., Sacramento, CA 95817 (USA)

The paper by Nazar et al., pp. 228-235 in this issue will generate considerable controversy. The singular lack of hard cutaneous and neurological findings and the extraordinary results of treatment of their patients beg many questions. One may argue that an identity crisis arises from their patient group who not only lack these preceding clinical features but also the typical neuroimaging and operative findings of the tethered cord syndrome. The decision to cut the filum is based on the first premise that there exists a group of children with some symptoms seen in tethered cord syndrome but lacking some of the hallmarks of the syndrome. The next premise is that neuroimaging studies on all patients and the electrophysiologi-cal data obtained on a few fail to show any abnormalities in the conus, nerve roots, meninges or filum. The final premise is that the sectioned filum is normal (radiologi-cally, operatively and histologically) but that inevitably the problem is in the filum.

As the authors state, a putative filum must exert its deleterious effect by producing abnormal tension on the conus. But this still boils down to a tethered conus by a nonresilient filum. A nonresilient filum may occur for developmental or acquired reasons. The developmental cause must involve some mis-step in secondary neurula-tion which also accounts for the cutaneous stigmata, hard neurological findings, musculoskeletal abnormalities, inclusions of fat, muscle, neuronal remnants and glia within a stout filum, distorted and elongated conus and concomitant dysraphic lesions such as lipomas and split cord malformations. Since none of the authors' patients have these characteristics of the developmental form of nonresilient filum, we must assume that their patients' fila are 'non-dysraphic'.

Is there then such a thing as an acquired nonresilient filum (which I personally have not encountered)? The authors believe that a filum can be injured during repetitive stretching ultimately rendering up a normal-looking but presumably stiff band. If this were the case, then why did none of their patients have a history of repetitive or catastrophic trauma to the lumbosacral spine? Why does this phenomenon not occur more often in adults, or athletes, gymnasts or combat personnel, who should have had more cumulative stretch trauma to their lumbosacral spine?

If neither a developmental nor an acquired cause for a nonresilient but normal-looking filum could be established, I find myself back to my initial intuition about this paper, i.e. that a filum which appears normal to all methods of observation is nothing more mysterious than a normal filum.

\author{
(C) 1996 S. KargerAG \\ Basel
}

